\title{
Tagungsbericht vom 50. Deutschen Historikertag 2014 in Göttingen: Wikipedia und Geschichtswissenschaft. Eine Zwischenbilanz
}

Die Erwartungen von Wikipedia und Geschichtswissenschaft aneinander sind nach wie vor sehr unterschiedlich. Während sich die einen mehr Beteiligung von Fachleuten erhoffen, um das Niveau ihrer Inhalte $\mathrm{zu}$ erhöhen, reiben sich die anderen an Qualität und Standards der Jedermanns-Enzyklopädie. Umso verdienstvoller war der Versuch, auf dem 50. Deutschen Historikertag beide Seiten im Rahmen einer Sektion zum Thema miteinander ins Gespräch zu bringen.

Die auf diesem Feld einschlägig positionierte Maren Lorenz (Toronto) präsentierte zum Auftakt einen kenntnisreichen und faktensatten Überblick - garniert mit bekanntem Skeptizismus (,Wikipedia. Ein Spiegel der Gesellschaft. Zum schwierigen Verhältnis von öffentlich verhandelter Geschichtsrepräsentation und -rezeption"). Allerdings erscheint fraglich, ob wissenschaftliche Standards im Sinne fachüblicher Peer-Review-Verfahren ein realistischer Maßstab für das MitmachLexikon sind. Hier könnte der Ansatz von Jan Hodel (Aarau) weiterführen, der in seinem Vortrag „Wikipedia. Geschichtsfragmente auf Abruf“ nach geeigneten Referenzsystemen Ausschau hielt. Ausgehend von einem intimeren Verständnis für die Wissensstrukturen, Beteiligungskulturen und Erwartungen der Nutzer/innen ließe sich möglicherweise ein reflektiertes Verhältnis der Geschichtswissenschaft zur Online-Enzyklopädie herstellen, die längst den Alltag der meisten Studierenden und auch vieler Forschender erobert hat. Auch Hodel beließ es jedoch in erster Linie bei einer Analyse der Veränderungen von Geschichtsrezeption und historischen Narrativen im Digitalen Wandel. Abgerundet wurde der erste, von Peter Hoeres (Würzburg) moderierte Teil der Sektion durch die Einblicke von Ziko van Dijk (Dortmund) in die Arbeit eines aktiven Wikipedianers („Wikipedia. Das ideale Hilfsmittel für den Einstieg in ein historisches Thema?“).

Unglücklicherweise war es bei der Organisation zu einem Missverständnis gekommen und die Veranstaltung nur als zweistündiger Block im Programmheft angekündigt worden. Daher verpasste ein Großteil der zu Beginn überaus zahlreich anwesenden über 130 Teilnehmer/innen den weitaus instruktiveren zweiten, von Georg Vogeler (Graz) geleiteten, Teil der Sektion, in dem es nun nicht mehr um die möglichen Probleme und Gefahren ging, sondern die drei Referenten erfreulich praxisorientiert über den Umgang mit der Wikipedia in Forschung und Lehre 
berichteten. Thomas Wozniak (Marburg) präsentierte eine Auswertung der Anzahl von entsprechenden Veranstaltungen in Vorlesungsverzeichnissen (83 zwischen 2006 und 2014) und Abschlussarbeiten (67 zwischen 2005 und 2013, darunter elf Dissertationen). Außerordentlich interessant waren auch seine Überlegungen zum Konzept kollaborativer Autorenschaft, das womöglich - neben der gewöhnungsbedürftigen Kommunikationskultur - eine der Hauptursachen für die Reserviertheit vieler Wissenschaftler/innen gegenüber der Online-Enzyklopädie sein könnte. Um Zitierfähigkeit zu gewährleisten, schlug er vor, bei einem Artikel-Anteil von 83 Prozent einzelner Hauptautor/innen oder von 70+13 Prozent im Falle zweier Hauptautoren/innen, die namentliche Zitierwürdigkeit festzulegen. Ähnlich argumentierte auch Andreas Kuczera (Gießen), der eine Befragung unter den Mitarbeiter/innen der Akademie der Wissenschaften und der Literatur in Mainz vorstellte. Demnach verfassen zwar nur wenige Seminarteilnehmer/innen selbst Artikel, doch hätten sich mittlerweile erstaunlich viele Geisteswissenschaftler/ innen daran gewöhnt, die Wikipedia als Quelle zu nutzen. Jürgen Nemitz (Marburg) referierte abschließend - verhalten optimistisch - über seine Erfahrungen in Seminaren an der Philipps-Universität Marburg, in denen er für den Leistungsnachweis das Verfassen eines Wikipedia-Artikels verlangt hatte.

Insgesamt ist es erstaunlich, wie wenig offizielle Auseinandersetzung es auch nach 14 Jahren noch gibt - trotz einzelner Annäherungsversuche. Die kulturellen Unterschiede zwischen den Communities sind wohl doch zu erheblich. Ob es jemals zu einer fruchtbaren, intensiven Zusammenarbeit kommen wird, scheint sehr fraglich. Mit der realen Existenz der Wikipedia und ihrer Nutzung für historische Zwecke wird sich die Geschichtswissenschaft aber dauerhaft arrangieren müssen.

\section{Sektionsübersicht}

Maren Lorenz (Toronto): Wikipedia. Ein Spiegel der Gesellschaft. Zum schwierigen Verhältnis von öffentlich verhandelter Geschichtsrepräsentation und -rezeption

Jan Hodel (Aarau): Wikipedia. Geschichtsfragmente auf Abruf

Ziko van Dijk (Dortmund): Wikipedia. Das ideale Hilfsmittel für den Einstieg in ein historisches Thema?

Peter Hoeres (Würzburg): Diskussion und Moderation

Thomas Wozniak (Marburg): Wikipedia in Forschung und Lehre. Eine Übersicht

Andreas Kuczera (Gießen): Wikipedia und Wissenschaftler - ein nicht repräsentatives Stimmungsbild

Jürgen Nemitz (Marburg): Wikipedia in der geschichtswissenschaftlichen Lehre. Bericht über Lehrveranstaltungen an der Philipps-Universität Marburg

Georg Vogeler (Graz): Diskussion und Moderation

Dieser Beitrag ist in veränderter Fassung auch veröffentlicht auf: Tagungsbericht: HT 2014: Wikipedia und Geschichtswissenschaft. Eine Zwischenbilanz, 23. 09.2014-26.09.2014 Göttingen, in: H-Soz-Kult, 04. 03. 2015, <http://www.hsozkult.de/conferencereport/id/ tagungsberichte-5868>. 